\title{
Clinical, etiological and progression factors of hearing in sudden deafness
}

\author{
Norma de Oliveira Penido ${ }^{1}$, Hugo Valter Lisboa \\ Ramos $^{2}$, Flávia Alencar Barros ${ }^{3}$, Oswaldo Laércio \\ Mendonça Cruz 4 , Ronaldo Nunes Toledo 5
}

Key words: sudden Deafness, sensorineural hearing loss.

\section{Summary}

$\mathrm{O}$ ut of the many forms of therapy for sudden deafness, some require hospitalization and present significant risks. Aim: This prospective study analyzes etiology and evolution in cases of sudden deafness (SD) where outpatient oral treatment was used. Study design: clinical with transversal cohort. Material and Method: Forty cases of sudden hearing loss were followed for at least one year. All were submitted to initial clinical evaluation, auditory tests, routine blood analysis, and magnetic resonance imaging. All received initial treatment with pentoxifylline and prednisone. Results: $45 \%(n=18)$ presented normal auditory thresholds, $40 \%$ $(n=16)$ showed some improvement in hearing, 15\% $(n=6)$ maintained initial hearing level. Nine cases $(22.5 \%)$ presented clinical conditions possibly implicated in hearing loss (viral infection, immunomediated hearing loss, vascular disorders, and so on); three (7.5\%) had cerebellopontine tumors. Evolution of hearing in these 12 cases with presumed etiology presented no differences from hearing in the 28 cases without any known etiological factor. Clinical treatment within the first seven days was the only statistically significantly different condition in patients who improved hearing. Conclusions: An objective search for etiological bases should be conducted in any case of acute sensorineural hearing loss. The presence of cerebellopontine tumors in $7.5 \%$ of cases of SD, among other treated causes, justifies a thorough clinical investigation in these patients. Overall good evolution of hearing was observed in $67.5 \%$ of cases of SD, regardless of its etiology. Therapy within the first seven days of SD was significantly related to better outcomes in hearing.

${ }^{1}$ Ph.D. in Medicine, Affiliated Professor, Unifesp-EPM.

${ }^{2}$ Master in Otorhinolaryngology, UNIFESP-EPM, Otorhinolaryngologist.

${ }^{3}$ Master in Health Sciences, UNIFESP-EPM, Speech and Hearing Therapist.

${ }^{4}$ Full Professor, Professor of the Department of Otorhinolaryngology, Federal University of Sao Paulo - Escola Paulista de Medicina. UNIFESP-EPM.

${ }^{5}$ Master in Otorhinolaryngology, UNIFESP-EPM, Otorhinolaryngologist. Department of Otorhinolaryngology, Federal University of Sao Paulo - Escola Paulista de Medicina. UNIFESP-EPM. Address correspondence to: Rua René Zanlutti 160 apt. 131 Chácara Klabin 04116-260 Sao Paulo SP. Article submited on May 16, 2005. Article accepted on September 12, 2005. 


\section{INTRODUCTION}

Sudden Deafness (SD) is defined as hearing loss greater than $30 \mathrm{~dB}$ in at least three subsequent frequencies of sudden onset or within maximum 72 hours, and it represents a common symptom to many different diseases and not a nosological entity in itself. In many situations, the etiology remains unknown and is a major challenge even after complete otological assessment. These cases are classified as idiopathic sudden deafness, and to those patients there is no consensus about what is the best treatment option or how the auditory recovery will be.

Different treatment approaches have been described, but most studies are not controlled or double blind studies ${ }^{1-}$ 5 . Moreover, spontaneous or placebo recovery rate is high and similar to results in patients treated with different drugs. Among the used drugs, corticoids seem to have universal acceptance and they are the only ones with confirmed efficacy ${ }^{1}$. It is also common to use drugs that reduce blood viscosity such as dextran or vasodilators such as carbogen., ${ }^{2,3}$ The use of antiviral therapies has increased, but the results are still not satisfactory. ${ }^{4,5}$ In fact, the different forms of treatment reflect the difficulties found in the treatment of a patient who does not have defined etiological diagnosis. Conversely, SD should be approached as an emergency, given that early intervention is associated with the best prognosis., 3

To contribute to the understanding of cases of SD and to promote better guidance in the cases of SD that were seen in our service, we conducted a prospective study for 2 years, analyzing the clinical and audiometric aspects, basic lab tests and imaging exams of these patients. The investigation of etiology of SD and the immediate observation of auditory progression regardless of the etiological diagnosis after the common initial treatment with prednisone and pentoxifylline were the main objectives of this study.

\section{MATERIAL AND METHOD}

The study included 40 patients who were seen at the Department of Otorhinolaryngology, Federal University of Sao Paulo - Escola Paulista de Medicina, Sao Paulo, with diagnosis of SD between the years 2000 and 2002. The criteria for inclusion in the study were sensorineural hearing loss greater than $30 \mathrm{~dB}$ in at least three subsequent frequencies of sudden onset or maximum within 3 days. To be included in the study we selected patients that had come to the center within 20 days from the installation of SD. All patients were submitted to a standard protocol for etiological investigation, they were also treated similarly and followed up for a minimum of 1 year.

Auditory assessment comprised pure tone and vocal audiometry, impedanciometry with acoustic reflex and speech recognition test. After the initial visit, the tests were repeated weekly in the first month, monthly up to the 6th month and then every six months up to discharge of the patient. Initial lab tests included complete blood count, fast glucose, total and fractioned cholesterol, triglyceride dosage and erythrocyte sedimentation rate.

In some situations there was complementation of the auditory assessment with distortion product otoacoustic emissions and electrocochleography and lab tests with dosage of proteins HSP 70 and $68 \mathrm{kD}$, FAN, dosage of complement and viral serology (mumps, herpes simplex types I and II, varicella zoster, cytomegalovirus, HIV and mononucleosis). Imaging exams comprised magnetic resonance imaging (MRI) of the encephalon, emphasis on temporal bone and posterior fossa. All the exams were preferably performed before or on the first days of treatment. We included in the study only the patients that performed a minimum follow up of one year and precisely complied with the clinical and diagnosis protocol.

Initial treatment was performed with prednisone $1 \mathrm{mg} / \mathrm{kg} /$ day, with tampering after 5 days and complete withdrawal at the end of three weeks, in association with pentoxifylline 400mg TID, which was maintained for 8 weeks. In patients who had etiological diagnosis of SD or presence of comorbidities (diabetes, hypertension, dyslipidemia) that required other drugs or approaches, they were used associated with standard therapy. All patients were treated in outpatient care.

The assessment of auditory recovery was performed through rate of improvement, which measured the perceptual auditory gain and used the contralateral side as a reference. $^{2}$

$\begin{aligned} \text { Rate of improvement }(\%)= & \text { Initial thresholds - Final } \\ & \text { thresholds X } 100 \\ & \begin{array}{l}\text { Initial thresholds - thresholds of } \\ \text { the contralateral ear }\end{array}\end{aligned}$

Auditory thresholds were calculated by using the means of frequencies 250, 500, 1000, 2000, 4000 and 8000 Hz. The final threshold was calculated based on the last audiometry, performed at least one year after the onset of treatment, and in all cases, auditory thresholds of the contralateral ear were below $30 \mathrm{~dB}$. Thus, it is evident that in our sample, all losses were unilateral. The improvement rates above $90 \%$ were defined as total recovery. Improvement rates below 20\% were considered therapeutic failure (no response). Improvement percentage between 21 and 89\% was defined as partial recovery, between 51 and 89\% the recovery was considered good, and if it remained between 21 and 50\%, it was considered fair.

The statistical analyses were performed using $\mathrm{X}^{2}$, Fischer and Analysis of Variance (ANOVA) tests to check the correlation between auditory recovery and age, gender, race, affected side, type of curve, presence of associated diseases, 
MRI affections or temporal bone and encephalon abnormalities, abnormal lab exams, concomitant symptoms, severity of hearing loss and beginning of treatment. p value should be below 0.05 to raise statistical significance.

To the statistical analysis, good results were the sum of cases that had total recovery plus those that had partial recovery and that presented improvement rate above 51\%. As dissatisfactory result, we considered the sum of those who had therapeutic failure plus those with recovery rate below $50 \%$.

\section{RESULTS}

Out of 40 patients, 22 (55\%) were male and 18 (45\%) were female and the mean age was 41 years, ranging from 13 to 76 years. The right ear was affected in $24(60 \%)$ and the left ear was affected in 16 (40\%) patients. In 33 (82.5\%) patients the hearing loss was instantaneous and in 7 (17.5\%) it was progressive, but it was completely within 72 hours. The presence of tinnitus was observed in 100\% of the cases. Vertigo or imbalance was detected in 21 patients $(52.5 \%)$ and ear fullness in 15 cases (37.5\%).

As to configuration of audiometric curve, flat losses reaching all frequencies were the most frequent ones, observed in 17 patients (42.5\%); ascending curves were observed in 10 cases (25\%), descending in $9(22.5 \%)$ patients, and in $4(10 \%)$ patients hearing loss affected preferably medium frequencies. As to severity of hearing loss at the diagnosis: 4 (10\%) patients presented mild loss, 15 (37.5\%) had moderate loss, 5 (12.5\%) had severe, and 16 (40\%) had profound loss.

The presence of associated diseases was found in 23 patients (57.5\%) and systemic hypertension and diabetes mellitus were found respectively in $9(22 \%)$ and 5 (12\%) patients as the most frequent diseases. All these findings were interpreted as comorbidities and not as cause of SD. A small increase in erythrocyte sedimentation rate was the most common lab test abnormality in the blood analysis of these patients.
Temporal bone and encephalon MRI identified affections to the central nervous system (CNS) or located in the inner ear in 12 patients (30\%). In the CNS, subcortical lesions compatible with microangiopathy were found in 7 cases (17.5\%). In 3 patients (7.5\%) we observed cerebellopontine angle tumors, and in one it was a meningioma (2.5\%) whereas in $2(5 \%)$ they were vestibular schwannomas. Inner ear hypersignal, identified at T1 after contrast, suggesting inflammatory activity, was found in 2 (5\%) patients. Microangiopathy was also considered as an associated factor and not as the cause of SD.

In 12 patients (30\%), a possible etiology of SD was defined (Chart 1): in 3 cases $(7.5 \%)$ it was an immunomediated disease with diagnosis based on lab findings, inflammatory activity observed in the inner ear at MRI or improvement and stabilization of hearing depending on the continuous administration of corticoids. Two (5\%) patients had vestibular schwannomas detected by MRI. Viral infection was observed in 2 cases (5\%), one with viral encephalitis and the second with mumps, the first confirmed through lumbar puncture and analysis of CSF and the second with parotid region edema, confirming the evaluation of IgM for mump virus and marked inflammatory activity in the inner ear observed at MRI. There were 2 other cases with vascular disorders, one with sickle cell anemia in an acute episode concomitant to the SD, and the other had SD and circulatory disorders that occurred during hemodialysis. Meningioma of cerebellopontine angle identified by MRI was seen in 1 patient (2.5\%). Ménière disease with vertigo and ear fullness occurred later during the follow up of the patient and it was confirmed through affection at SP/AP ratio in electrocochleography found in 1 patient $(2.5 \%)$. One patient $(2.5 \%)$ had SD caused by barotrauma, observed immediately after physical exercises at the gym.

During the follow up period, 18 patients (45\%) presented normal hearing, that is, recovery rate above $90 \%$ and $9(22.5 \%)$ presented partial improvement, with recovery rates between $51 \%$ and $89 \%$, amounting to a total of 27 $(67.5 \%)$ patients with satisfactory progression. The total

Chart 1. Possible etiology of sudden deafness.

\begin{tabular}{|cccc|}
\hline No & Etiology & Rate of improvement & Diagnosis \\
\hline 01 & Rupture of membrane & 100 & History \\
02 & Schwannoma & 100 & MRI \\
03 & Vascular & 100 & History / Lab tests \\
04 & Immunomediated & 100 & Lab tests \\
05 & Meningioma & 100 & MRI \\
06 & Viral & 100 & History /Lab tests \\
07 & Immunomediated & 51 & History / Lab tests \\
08 & Méniere & 45 & History/Lab tests \\
09 & Vascular & 38.5 & History \\
10 & Immunomediated & 36.6 & Progression / Lab tests \\
11 & Schwannoma & 8.6 & MRI \\
12 & Viral & 0 & History/Lab tests \\
\hline
\end{tabular}


number of cases with dissatisfactory progression was 13 (32.5\%) patients, being 6 (15\%) without responses, whose rates of auditory recovery were up to $20 \%$, and 7 (17.5\%) with poor response, with recovery rates between 21 and 50\% (Graph 1).

It is interesting to observe that auditory recovery in statistical terms between the patients with defined etiological diagnosis and idiopathic cases of SD was similar (Fischer test, $\mathrm{p}=0.195$, Graph 2). Other analyzed clinical aspects with the possible relation in auditory recovery were age, gender, race, involved side, audiometric configuration, initial level of hearing loss, concomitant symptoms, associated diseases, MRI observed affections and lab tests that did not statistically differ in auditory recovery. The exception was observed in time of beginning of treatment, because the patients that started treatment with prednisone and pentoxifylline in the first 7 days after onset of SD had better auditory recovery when compared to those that started treatment later $(\mathrm{p}<0.05)$ (Graph 3).

\section{DISCUSSION}

When we discuss SD, the terminology should be well defined, given that there is no uniformity about this topic in the literature. In some situations, SD may refer only to cases in which there is sudden sensorineural loss of idiopathic origin, but, in most cases, we are referring to all forms of sensorineural loss of sudden manifestation, regardless of the etiological diagnosis.

Another aspect to be considered is the method employed to assess auditory recovery in SD. Different criteria are used, some assess absolute gain in decibel (dB), calculating the difference between final and initial audiometry, without taking into account the severity of hearing loss. The failure in this analysis is that the gain of 20 $\mathrm{dB}$ in a patient with a mild or moderate loss has completely different functional meaning when compared to profound loss. Another form normally used to analyze the results is the investigation of the so-called relative auditory gain, which is calculated by the percentage of improvement between final and initial audiometry of the patient. In this situation, the analysis of auditory recovery is more reliable, given that the percentage value has the same weight regardless of the hearing loss severity. In our study, we used the rate of auditory improvement which, in addition to analyzing the percentage of recovery of the affected ear, considers the contralateral hearing as a reference. According to these criteria, the patient is classified in an increasing scale of improvement and not in static groups, in which the intragroup variation is very high. It represents the best parameter to assess auditory recovery and predicts efficacy of treatment. ${ }^{7}$

The percentage of spontaneous improvement ranges, according to the literature, from 45 to $65 \% \%^{7,8}$ and available

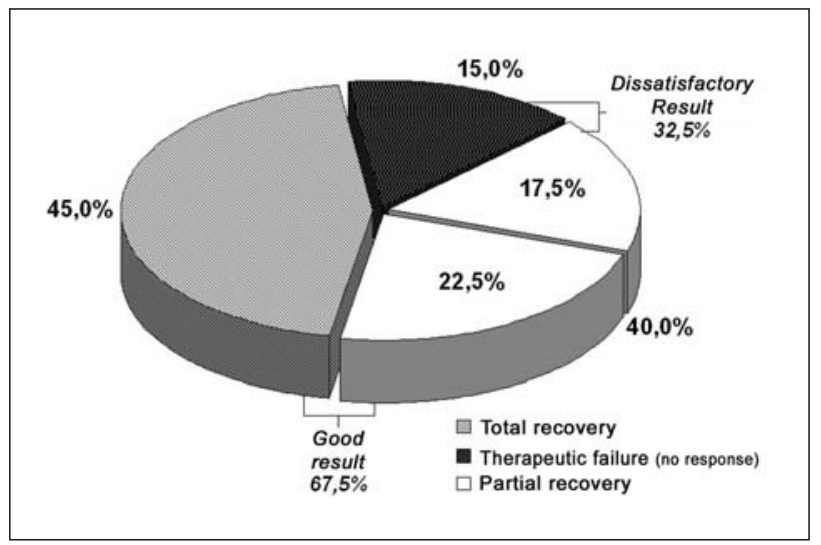

Graph 1. Rate of auditory improvement after 1 year.

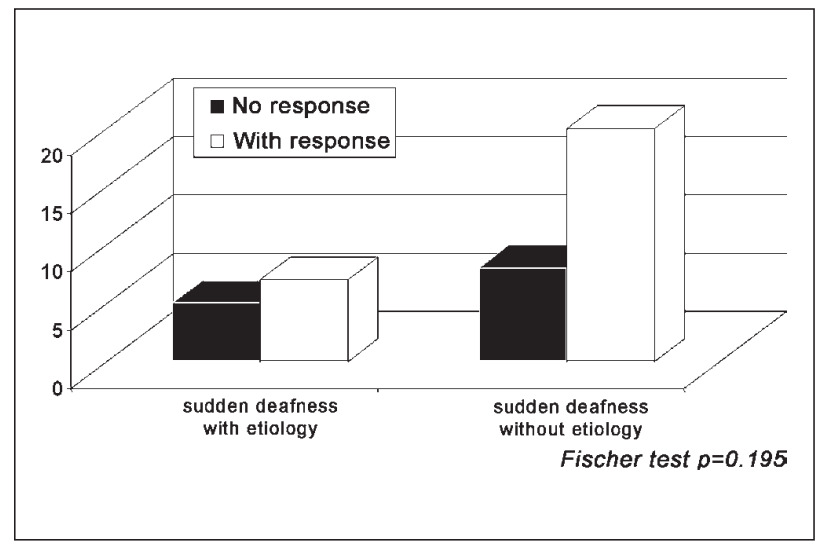

Graph 2. Etiological diagnosis and auditory recovery.

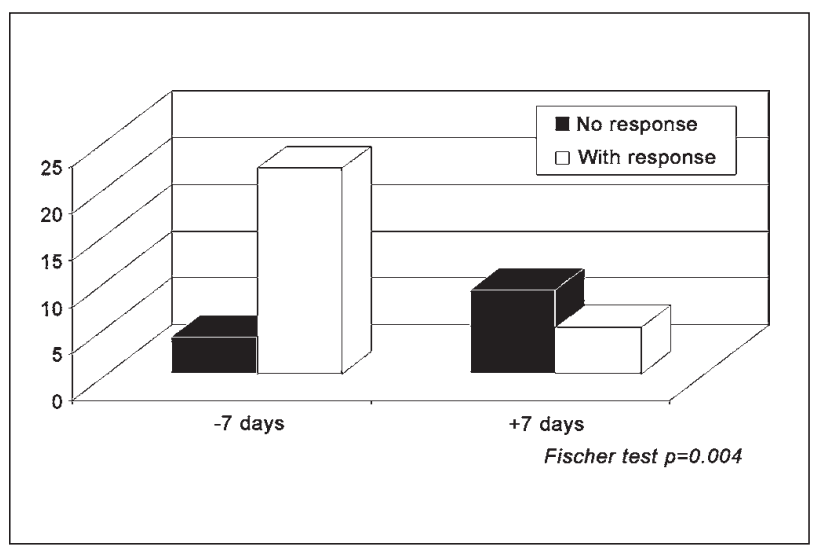

Graph 3. Time from onset of treatment and auditory improvement. 
treatment options present similar results. In part, this percentage variation occurs owing to more or less flexibility in the use of improvement criteria. In studies about gain of $10 \mathrm{~dB}$ in relation to initial audiometry we consider it an improvement, and the rates tend to come close to $80 \% .{ }^{4}$ In our study, we only considered complete auditory improvement when the recovery rates were over $90 \%$ and had significant improvement when they were over 50\%, much more rigorous values than those described in the literature ${ }^{3}$. Even so, by adding up the two groups, the improvement rate observed was $67.5 \%$, which is similar to the rate described by other authors with other therapies. ${ }^{3,8-}$ 10

In our study, we used a previously defined protocol in all patients with SD that came to the Emergency room, and diagnosis of etiology was found in 30\% of the cases. Other studies reported identification of etiology in only 10 to $15 \%$ of the cases ${ }^{6,9}$. These differences may be justified by the use of different clinical protocols that may have different sensitivity levels.

In our study, we found cerebellopontine angle tumors in $7.5 \%$ of the cases, findings that were higher than what was reported in the literature 5 . It was even more interesting to perceive that vestibular schwannoma presented total recovery of hearing after the advocated initial treatment. This fact had been described before ${ }^{5}$, and auditory recovery could interfere in the choice of surgical access, influencing the auditory prognosis of these patients.

$\mathrm{SD}$ treatment is another topic that generates much discussion. A wide range of therapeutic modalities may be found in the literature, some are complex and uncommon, but they have the same efficacy. Most treatment approaches are based on a possible circulation disorder or inflammatory reaction that affects the inner ear ${ }^{8}$. However, histological analyses of the cochlea of patients who had SD presented affections compatible with viral lesions. ${ }^{11,12}$ Despite these histological evidences and experimental studies demonstrating the efficacy of antiviral in the treatment of $\mathrm{SD}^{13}$, the beneficial effects of antiviral in humans have not been confirmed yet. ${ }^{4,5}$ Wide spectrum therapies that would theoretically treat both vascular and inflammatory disorders are widely used in clinical practice; in part, because there are no treatments with recognized efficacy and also owing to the anxiety that involves the physician-patient relationship in these cases. However, there is no evidence that this type of approach has produced better results than monotherapy.

There are few controlled studies in the literature and only corticoids seem to have actually shown any benefit in the treatment of SD. ${ }^{1,9,14}$ As to vasodilators, even though they are widely used, there is still scarcity of studies with more rigid scientific controls that confirm their positive effects in the treatment of SD. ${ }^{9}$ In fact, the attempts to confirm the increase in cochlear blood flow with the use of these drugs have been inconsistent. ${ }^{10}$ Pentoxifylline, a medication with hemorheological effects, reduces blood viscosity improving the blood flow in the labyrinth and, as previously described, it presents beneficial effects in patients with SS. ${ }^{15}$ The option to use prednisone associated with pentoxifylline in our study was a result of the fact that both can be used as outpatient drugs, preventing the inconvenience of hospitalization and minimizing the potential risks of the use of intravenous vasodilators or corticoids.

The purpose of our study was not compare different therapies. Our focus was on the etiological identification of SD and the follow up of immediate progression of these patients after common therapy with pentoxifylline and prednisone.

As to demographical characteristics, our results were similar to most of those described in the literature, with mean age of patients in the 5 th decade of life and with no predominance of side or gender. ${ }^{3,4,6}$

Differently from other authors, we did not find any correlation between type of audiometric curve or severity of hearing loss in auditory recovery ${ }^{4}$. There were patients with mild losses that did not recover hearing and others with profound loss who fully recovered it. There was predominance of audiometric curves of flat configuration, which presented similar losses in all frequencies. Ascending curves or those with predominance of medium frequencies did not show better prognosis in our sample than what was described in the literature. $^{6}$

The presence of associated symptoms, such as tinnitus and vertigo, was related with worse prognosis by many authors ${ }^{3,4,16}$. In our sample, vestibular symptoms of vertigo and imbalance were present in $52.5 \%$ of the patients, but the presence of tinnitus was greater than what had been reported by other authors ${ }^{3,4}$, affecting at the beginning of the presentation $100 \%$ of the patients. However, none of the symptoms presented any correlation with degree of auditory recovery in the study.

The presence of concomitant diseases occurred in $60 \%$ of the cases and the presence has also not affected the auditory prognosis of the study. Given that mean age of the patients was high, the presence of concomitant diseases was correlated with their own characteristics, more than with SD. The frequent finding of microangiopathy in CNS observed at MRI helped us confirm this fact given that diabetes mellitus and hypertension were the most prevalent diseases in the groups of patients. This finding was not considered an etiological factor of SD.

The only statistically significant prognostic factor was early onset of therapy. The patients that were seen within 1 week from onset of condition presented better hearing recovery rates, a similar finding to that of other authors ${ }^{3,6}$. The initial analysis of this piece of information could suggest that treatment is more effective than natural progression of the disease. However, given that SD may quickly recover, some patients with this type of progression do not come to 
the medical center and, thus, the finding of better progression in the case of early introduction of the drug could represent one more effect of natural progression of SD rather than the treatment per se. In the group that started treatment more than 1 week after onset we can include most of the patients that did not present spontaneous improvement, explaining the difference in auditory recovery between the two groups. Only a double blind and placebo-controlled study could separate the natural progression from good results with the adopted treatment. However, the studies with this methodological design are difficult to perform for obvious ethical issues.

When we compare auditory progression between patients with idiopathic SD and those in which etiological diagnosis was made, we did not observe statistically significant differences between them, despite the more directed treatment to patients with specific etiology. This observation shows that despite the diagnostic advances obtained with the routine performance of new laboratory exams or imaging exams in patients with SD, which have reduced the number of idiopathic cases, the therapeutic success of the attempt to improve auditory function of these patients is still limited, despite the possibility of performing a more specific treatment, especially in cases associated with viral diseases.

\section{CONCLUSION}

The objective investigation of etiology should be performed in any case of SD. The presence of cerebellopontine tumors in $7.5 \%$ of the cases of SD, among other causes, justifies complete investigation in these patients. Good progression, with improvement of $67.5 \%$ of the cases of SD, was observed regardless of the etiological diagnosis. The beginning of therapy within the first week from onset was the only factor related with better auditory results in these patients.
2. Shiraishi T, Kubo T Okumura S, Naramura H, Nishimura M, Okusa $\mathrm{M}$, Matsunaga T. Hearing recovery in sudden deafness patients using a modified defibrinogenation therapy. Acta Otolaryngol Suppl 1993, 501: 46-50.

3. Gordin A, Goldenberg D, Golz A, Netzer A, Joachims HZ. Magnesium: A new therapy for idiopathic sudden sensorineural hearing loss. Otology \& Neurotology 2002; 23: 447-51.

4. Stokroos RJ, Albers FW, Tenvergert EM. Antiviral treatment of idiopathic sudden sensorineural hearing loss: A prospective, randomized, double-blind clinical trial. Acta Otolaryngol 1998; 118: 488-95.

5. Tucci DL, Farmer Jr, JC, Kitch RD, Witsell DL. Treatment of sudden sensorineural hearing loss with systemic steroids and valacyclovir. Otology \& Neurotology 2002; 23: 301-8.

6. Zadeh MH, Storper IS, Spitzer JB. Diagnosis and treatment of sudden-onset sensorineural hearing loss: A study of 51 patients. Otolaryngol Head Neck Surg 2003; 128 (1): 92-8.

7. Yamamoto M, Kanzaki J, Ogawa K, Ogawa S, Tsuchihashi N. Evalution of hearing recovery in patients with sudden deafness. Acta Otolaryngol Suppl 1994, 514: 37-40.

8. Mattox DE, Simmons FB. Natural history of sudden sensorineural hearing loss. Ann Otol Rhinol Laryngol 1977; 86 (4 pt1): 463-80.

9. Haberkamp TJ, Tanyeri HM. Management of idiopathic sudden sensorineural hearing loss. Am J Otol 1999; 20 (5): 587-95.

10. Ohlsen KA, Didier A, Baldwin D, Miller JM, Nuttal AL, Hultcrantz E. Cochlear blood flow in response to dilating agents. Hear Res 1992; 58 (1): 19-25.

11. Schuknecht HF, Donavan ED. The pathology of idiopathic sudden sensorineural hearing loss. Arch Otorhinolaryngol 1986; 243 (1): $1-15$.

12. Schuknecht HF. Myths in neurotology. Am J Otol 1992; 13 (2): 124-6.

13. Stokroos RJ, Albers FW, Schirm J. Therapy of idiopathic sudden sensorineural hearing loss: Antiviral treatment of experimental herpes simplex virus infection of the inner ear. Ann Otol Rhinol Laryngol 1999, 108: 423-8.

14. Wilson WR. Why treat sudden haring loss. Am J Otol 1984, 5 (6): 481-3.

15. Hormann K. Rheological effects of pharmacological treatment of inner ear disorders. Scand Audiol Suppl 1987; 26: 47-8.

16. Byl FM. Sudden hearing loss: eight years' experience and suggested prognostic table. Laryngoscope 1984; 94 (5 pt 1): 647-61.

\section{REFERENCES}

1. Wilson WR, Byl FM, Laird N. The efficacy of steroids in the treatment of idiopathic sudden hearing loss. A double-blind clinical study. Arch Otolaryngol 1980, 106(12): 772-6. 